Suppression of Type-I ELMs Using a Single Toroidal Row of Magnetic Field Perturbation Coils in DIII-D

M. E. Fenstermacher, T. E. Evans, T. H. Osborne, M. J. Schaffer, J. S. deGrassie, P. Gohil, R. A. Moyer

March 20, 2008

Nuclear Fusion 
This document was prepared as an account of work sponsored by an agency of the United States government. Neither the United States government nor Lawrence Livermore National Security, LLC, nor any of their employees makes any warranty, expressed or implied, or assumes any legal liability or responsibility for the accuracy, completeness, or usefulness of any information, apparatus, product, or process disclosed, or represents that its use would not infringe privately owned rights. Reference herein to any specific commercial product, process, or service by trade name, trademark, manufacturer, or otherwise does not necessarily constitute or imply its endorsement, recommendation, or favoring by the United States government or Lawrence Livermore National Security, LLC. The views and opinions of authors expressed herein do not necessarily state or reflect those of the United States government or Lawrence Livermore National Security, LLC, and shall not be used for advertising or product endorsement purposes. 


\title{
Suppression of Type-I ELMs Using a Single Toroidal Row of Magnetic Field Perturbation Coils in DIII-D
}

\author{
M.E. Fenstermacher, ${ }^{\text {a) }}$ T.E. Evans, ${ }^{\text {b) }}$ T.H. Osborne, ${ }^{\text {b) }}$ M.J. Schaffer, ${ }^{\text {b) }}$ J.S. deGrassie, ${ }^{\text {b) }}$ P. \\ Gohil, ${ }^{\text {b) }}$ R.A. Moyer, ${ }^{\text {c) }}$ and the DIII-D Team \\ ${ }^{a)}$ Lawrence Livermore National Laboratory, Livermore, California, USA

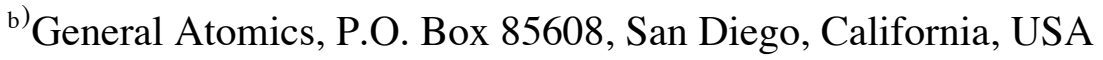 \\ ${ }^{c}$ University of California-San Diego, La Jolla, California, USA
}

\begin{abstract}
In recent DIII-D [J.L. Luxon, et al., Nucl. Fusion 43, 1813 (2003)] experiments, suppression of Type-I Edge Localized Modes (ELMs) was obtained by applying $n=3$ Resonant Magnetic Perturbations (RMPs) using a single toroidal row of internal, small aperture coils located either above or below the equatorial plane in plasmas with ITER-like electron pedestal collisionality $v_{\mathrm{e}}^{*} \sim 0.1$, flux surface shape and low edge safety factor $\left(q_{95} \approx 3.6\right)$. The single-row of internal RMP coils achieved suppression at approximately the same surface-averaged magnetic field perturbation strength at the $q_{95}$ surface as needed for suppression with two-rows of poloidally separated coils, but this required higher current per coil than needed for suppression with two-rows of coils. Suppression of Type-I ELMs was not obtained with similar $n=3$ perturbation strength at the $q_{95}$ surface from a single-row of external, large aperture coils on the outer equatorial midplane.
\end{abstract}




\section{INTRODUCTION}

Control of Edge Localized Mode (ELM) instabilities in high confinement (H-mode) tokamak plasmas is a critical issue for the operation of future high performance tokamaks including ITER [1] due to predictions of unacceptably high erosion of material surfaces by the heat and particle fluxes during these transient events. Various techniques to reduce ELM size or eliminate ELMs all together are under investigation (see Ref 2 and references therein). Many experiments in DIII-D have shown that ELMs can be suppressed in H-mode plasmas over a wide range of conditions (density, collisionality, input power, safety factor etc.) by applying $n=3$ Resonant Magnetic Perturbations (RMPs) using two toroidal rows of internal, small aperture magnetic coils poloidally separated above and below the outer equatorial midplane (Ref 2 and references therein). Recent experiments described below achieved ELM suppression for the first time with $\mathrm{n}=3$ RMPs using only one of the internal, toroidal, off-midplane rows. In contrast, ELMs were not suppressed with $n=3$ RMPs of comparable amplitude using a single row of external, large aperture coils centered on the equatorial midplane. 


\section{EXPERIMENTAL RESULTS}

For the experiments reported in this paper the plasmas had an ITER Similar Shape (ISS) and pedestal electron collisionality close to the value expected in ITER, $v_{\mathrm{e}}^{*} \sim 0.1$. The plasma configuration and the geometry of the internal, off-midplane (I-coil) and external, on-midplane (C-coil) magnetic perturbation coils were the same as reported in Ref. 2 (see Fig. 1 therein). Operating parameters in the present experiments were: plasma current $I_{\mathrm{p}}=1.55 \mathrm{MA}$, toroidal field $B_{\mathrm{T}}=1.93 \mathrm{~T}$, lower triangularity $\delta_{\text {lower }}=0.67-0.70$, upper triangularity $\delta_{\text {upper }}=0.36$, elongation $\kappa=1.82$, giving safety factor at $95 \%$ poloidal flux, $q_{95}=3.47-3.62$. Injected neutral beam power was $P_{\text {inj }}=$ 7.1-9.5 MW, giving normalized beta $\beta_{\mathrm{N}}=\beta /\left(I_{\mathrm{p}} / a B_{\mathrm{T}}\right)=1.7-2.4$, where $a$ is the minor radius of the plasma and $\beta$ is the ratio of plasma pressure to magnetic field pressure.

Suppression of Type-I ELMs was achieved using a single toroidal row of small aperture, off-midplane internal coils but more current per coil was required than for suppression with two rows of internal coils (Fig. 1). ELM suppression was obtained with 4.5 kAt per coil when using only the upper I-coil row (giving a surface-averaged $n=3$ resonant radial vacuum field in the $m / n=11 / 3$ component $\delta b_{r}^{11 / 3}=4.0 \mathrm{G}$ at $\Psi_{N}=0.95$; Fig. 1f) and at $4.8 \mathrm{kAt}$ with only the lower I-coil row $\left(\delta b_{r}^{11 / 3}=4.2 \mathrm{G}\right.$ at $\Psi_{N}=0.95$; Fig. 1g). For reference, ELM suppression was obtained in a similar discharge (Fig. 1h) using both the upper $(2.9 \mathrm{kAt})$ and the lower $(2.5 \mathrm{kAt}) \mathrm{I}$-coil rows $\left(\delta b_{r}^{11 / 3}=4.6 \mathrm{G}\right.$ at $\left.\Psi_{N}=0.95\right)$ i.e., the conventional configuration with $\mathrm{n}=3$ even parity RMPs. Note also that in the comparison of Fig.1, ELM suppression was obtained with both I-coil rows at the same perturbation strength even though the pedestal density was higher than in the single row cases (Fig. 1c). At this higher density, a scan of the current in the single upper I-coil row Figs. 1(d) and (e) shows the minimum current required to suppress ELMs was between 4.0 and $4.5 \mathrm{kAt}$ (ie. $\left(\delta b_{r}^{11 / 3}=3.5-4.0 \mathrm{G}\right.$ at $\left.\Psi_{N}=0.95\right)$, ie. at least $50 \%$ higher 
current per coil was required to achieve similar perturbation strength at the pedestal than when both I-coil rows are used, consistent with the difference in coil geometry. Comparison of the poloidal mode spectra, at comparable density, for the case with the upper I-coil vs. the case with both I-coils (Fig. 2) shows comparable perturbation strength in the pedestal region, $0.95<\Psi_{N}<1.0$, but significantly different resonant and nonresonant components outside the pedestal. 
ELM suppression with a single-row of internal coils requires more NBI power and/or co-torque than suppression with two rows of internal coils. In three comparable discharges with different timing of an increase from 7.1 to 9.5 MW of injected NBI power, ELM suppression was obtained only after the increase to the higher power (Fig. 3). This implies that the required minimum co-NBI power for suppression with the upper I-coil alone was between 7.1 and $9.5 \mathrm{MW}$ of co-injected power, providing between 4.85 and $6.8 \mathrm{~N}$-m of co-torque. In contrast, ELM suppression with two rows of I-coils has been obtained previously (3) in plasmas with $\sim 4.0 \mathrm{MW}$ of co-NBI $(2.8 \mathrm{~N}-\mathrm{m}$ of cotorque). Figs. 3d vs 3e show the clearest example using the upper I-coil row in which ELMs persist at reduced amplitude after the RMP is turned on at the 7.1 MW power level. ELM suppression in both cases is obtained within 50-100 ms after the increase to the 9.5 MW power level. Comparing Figs. 3d and 3f shows that ELM suppression is achieved about $150 \mathrm{~ms}$ earlier in the discharge with $9.5 \mathrm{MW}$ injected power prior to the Icoil turn-on time. Fig. $3 \mathrm{c}$ also shows that the pump-out of the pedestal density at the Icoil turn-on is similar in the three discharges suggesting less sensitivity of the change in particle transport to the power level than for ELM suppression.

Experiments using $\mathrm{n}=3$ fields from a single toroidal row of coils with 6 loops located on the equatorial plane external to the DIII-D vacuum vessel (the DIII-D C-coil) were not successful in suppressing ELMs at coil currents sufficient to produce the same level of field perturbation in the pedestal as in the cases with internal coils. A comparison of cases using the upper I-coil and the C-coil (Fig. 4) shows no reduction in the maximum ELM size in the C-coil case (Fig. 4f). The I-coil case used n=3 RMP currents of $4.5 \mathrm{kAt}$ and $6.0 \mathrm{kAt}\left(\delta b_{r}^{11 / 3}=4.0\right.$ and $5.3 \mathrm{G}$ respectively at $\left.\Psi_{N}=0.95\right)$ with the C-coil providing optimum n=1 error field correction (EFC). The C-coil discharge used n=3 RMP currents of $15 \mathrm{kAt}$ and $18 \mathrm{kAt}\left(\delta b_{r}^{11 / 3}=3.8\right.$ and $4.6 \mathrm{G}$ respectively at $\left.\Psi_{N}=0.95\right)$ with the I-coil providing optimum n=1 EFC. ELMs were suppressed with the upper I-coil at the $4.5 \mathrm{kAt}$ $\left(\delta b_{r}^{11 / 3}=4.0 \mathrm{G}\right.$ at $\left.\Psi_{N}=0.95\right)$ perturbation level but ELMs remained with the C-coil up to 
$18 \mathrm{kAt}\left(\delta b_{r}^{11 / 3}=4.6 \mathrm{G}\right.$ at $\left.\Psi_{N}=0.95\right)$. Here the pedestal density (Fig. $\left.4 \mathrm{~d}\right)$ is similar between the two discharges for about $150 \mathrm{~ms}$ prior to the RMP turn-on although the density evolution earlier in the discharges is different due to a locked mode induced brief return to L-mode in the case with the upper I-coil. At the RMP turn-on, pedestal density pumpout is observed in the case with the upper I-coil but little evidence of pumpout is seen in the $\mathrm{C}$-coil case. Initial attempts to increase the $\mathrm{n}=3 \mathrm{C}$-coil RMP current further resulted in locked modes with no indication of reduction in ELM size. 


\section{DISCUSSION OF THEORY - EXPERIMENT COMPARISON}

The qualitative differences between ELM suppression with internal, off-midplane coils and the lack of suppression with similar $n=3$ perturbation strength from external onmidplane coils is consistent with the model of a minimum required island overlap width [2] from mode spectrum analysis [4]. A comparison of vacuum field perturbation island structures and the profiles of the Chirikov parameter (Fig. 5) for the plasmas of Fig. 4 shows that the overlap region with Chirikov parameter $>1.0$ is larger in the case that achieved suppression with the upper I-coil. In addition, the upper I-coil case has good overlap of the $(3 / 1,6 / 2,9 / 3)$ structures at the $q=3$ surface and the adjacent $10 / 3$ island farther out in the pedestal, but there is a gap between the $(3 / 1,6 / 2,9 / 3)$ and $10 / 3$ islands in the C-coil case. The other cases of ELM suppression with either the upper or lower Icoils alone, or with both I-coils together, also show good overlap of the $(3 / 1,6 / 2,9 / 3)$ island and the remaining islands out to the plasma edge. This qualitative difference in the ELMing case with the C-coil RMP is consistent with the hypothesis from Ref. 2 that coupling of the remnant $7 / 3,5 / 2$ and $8 / 3$ island structure adjacent to a highly stochastic region from the $\mathrm{q}=3$ surface to the edge of the plasma may play an important role in achieving suppression of ELMs in DIII-D. 


\section{CONCLUSIONS}

Suppression of Type-I ELMs was achieved for the first time with a single toroidal row of internal, small aperture, off-midplane, $n=3$ RMP coils in DIII-D but was not achieved with the same field perturbation strength at the pedestal from a single row of external, large aperture, on-midplane n=3 RMP coils. Higher current per coil (consistent with the difference in geometry) and higher injected NB power or co-injected torque was required to suppression ELMs in the cases with a single toroidal row compared with suppression using toroidal rows of internal coils above and below the midplane simultaneously. The results appear to be consistent with qualitative differences in initial mode spectral analysis of the vacuum perturbation fields and a model [2] proposing a required minimum width of the edge region having good overlap of the magnetic islands to achieve ELM suppression. 


\section{Acknowledgment}

This work was performed under the auspices of the U.S. Department of Energy by Lawrence Livermore National Laboratory in part under Contract W-7405-ENG-48 and in part under Contract DE-AC52-07NA27344, and under DE-FC02-04ER54698, DE-FG0207ER54917 and DE-FG02-05ER54809. 


\section{References}

1. ITER Physics Basis Editors, “ITER Physics Basis,” Nucl. Fusion 39, 2137 (1999); ITER Physics Basis Editors, "Progress on the ITER Physics Basis," accepted for publication in Nucl. Fusion (2007).

2. M.E. Fenstermacher, T.E. Evans, T.H. Osborne, M.J. Schaffer, et al., "Effect of island overlap on ELM suppression by resonant magnetic perturbations in “ DIII-D,” Phys. Plasmas, in press (2008)

3. K. H. Burrell, T. E. Evans, E. J. Doyle, et al., Plasma Phys. Control. Fusion 47, B37 (2005).

4. M.J. Schaffer, J. E. Menard, M. P. Aldan et al., NF 48, 024004 (2008) 


\section{Figure Captions}

Fig. 1. Comparison of parameters from plasmas with ELMs suppressed using the upper I-coil alone (black-circle, red-square, blue-diamond), the lower I-coil alone (majentatriangle) and both I-coils simultaneously (green-cross) including (a) plasma current (MA) and injected co-current neutral beam power (MW), (b) I-coil current (kAt), (c) pedestal electron density $\left(10^{19} \mathrm{~m}^{-3}\right)$, and (d-h) outer divertor $D_{\alpha}$ intensity $\left(1 \times 10^{17} \mathrm{phot} / \mathrm{m}^{2} / \mathrm{s} / \mathrm{str}\right)$. Cases with the upper I-coil alone include (d) an ELMing plasma at $4.0 \mathrm{kAt}$ and high density, (e) an ELM suppressed plasma at $4.5 \mathrm{kAt}$ at moderately high density comparable to the case with both I-coils and (f) an ELM suppressed plasma at $4.5 \mathrm{kAt}$ at the same low density as in the case (g) with the lower I-coil alone.

Fig. 2. Comparison of the radial component of the $n=3$ helical mode spectra using vacuum fields [4] for the ELM suppressed plasmas from (a) Fig. 1e at 3000ms (upper Icoil alone at $4.5 \mathrm{kAt}$ ) and (b) Fig. 1(h) at 2650ms (upper and lower Icoils at 2.9 and 2.5 kAt respectively). Pitch resonant modes with $m=-n q\left(\Psi_{\mathrm{N}}\right)$ are shown by the white dashed line.

Fig. 3. Comparison of the same parameters as in Fig. 1 from plasmas using $4.5 \mathrm{kAt}$ in the upper I-coil alone with early (blue-diamond), intermediate (black-circle), and late (redsquare) timing of the increase in the injected neutral beam power from 7.1 to $9.5 \mathrm{MW}$.

Fig. 4. Comparison of parameters from plasmas with ELMs suppressed using the upper I-coil alone (black-circle), ELMs using the C-coil n=3 RMP (red-square) including (a) plasma current (MA) and injected co-current neutral beam power (MW), (b) C-coil current (kAt), (c) I-coil current (kAt), (d) pedestal electron density $\left(10^{19} \mathrm{~m}^{-3}\right)$, and (e-f) outer divertor $D_{\alpha}$ intensity $\left(1 \times 10^{17} \mathrm{phot} / \mathrm{m}^{2} / \mathrm{s} / \mathrm{str}\right)$. 
Fig. 5. Comparison of RMP vacuum island structure and Chirikov parameter profile [4] for (a) the ELM suppressed discharge using the upper I-coil alone (Fig. 4 black traces) at $3000 \mathrm{~ms}$ and (b) the ELMing discharge using the C-coil at comparable perturbation amplitude in the pedestal (Fig. 4 red traces) at $4000 \mathrm{~ms}$. Island widths are aligned with their resonant q-value for $n=1$ (black), $n=2$ (green) and $n=3$ (red) as in Ref. 2. Vertical dashed line indicates width of region with Chirikov parameter $>1.0$. 


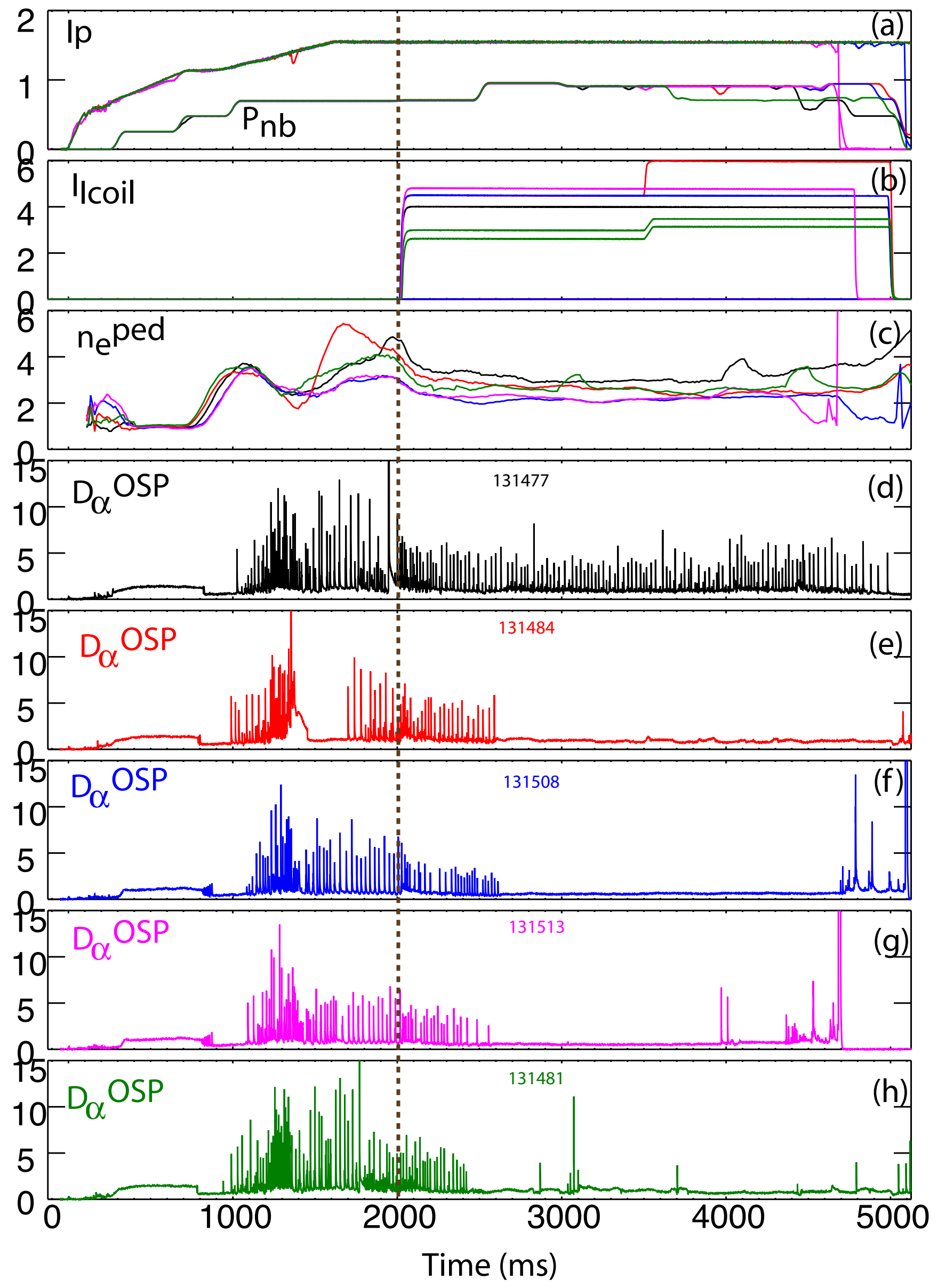

Fenstermacher_NF_Lett08_Fig.1new 


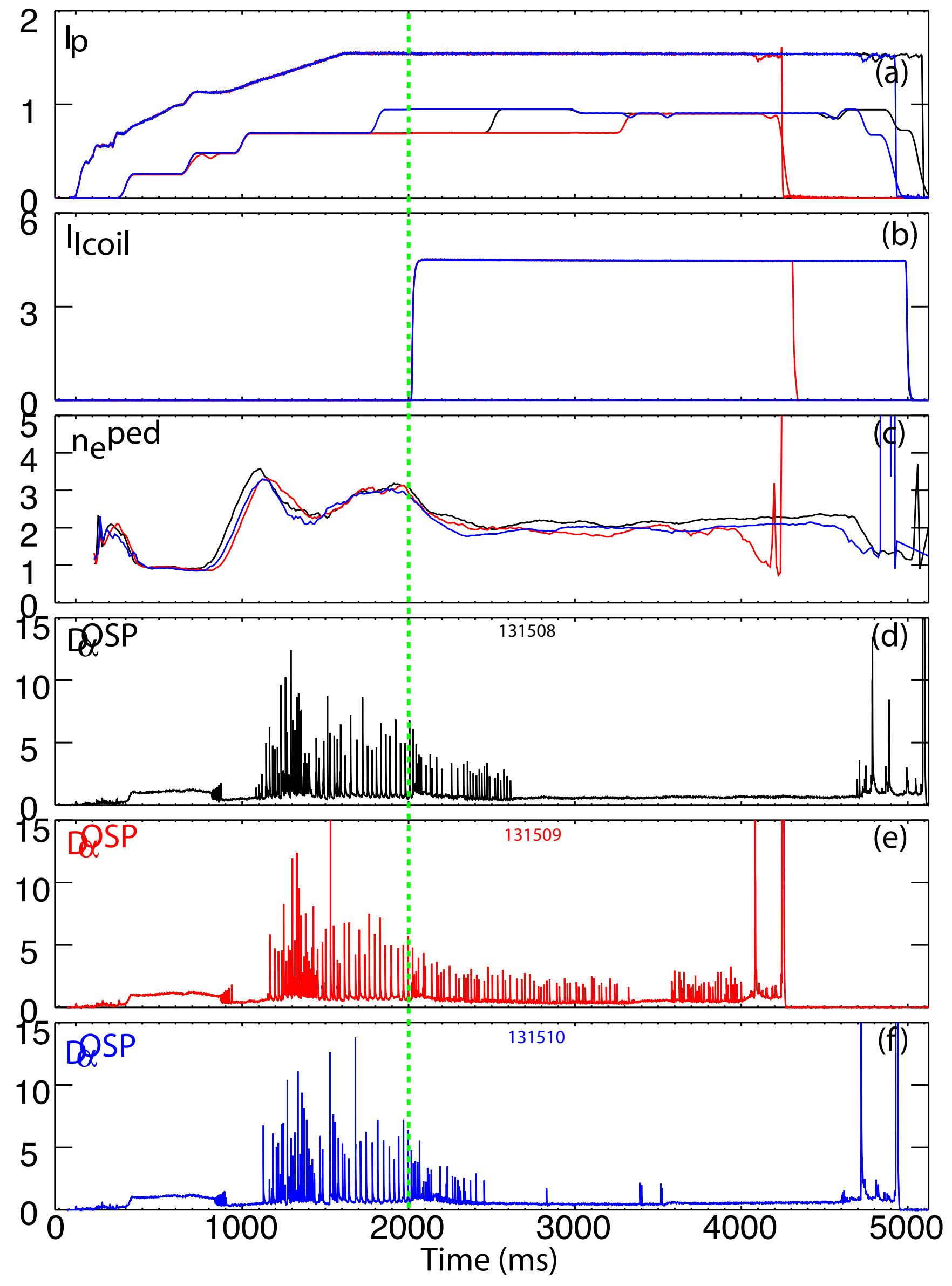

Fenstermacher_NF_Lett08_Fig.3 


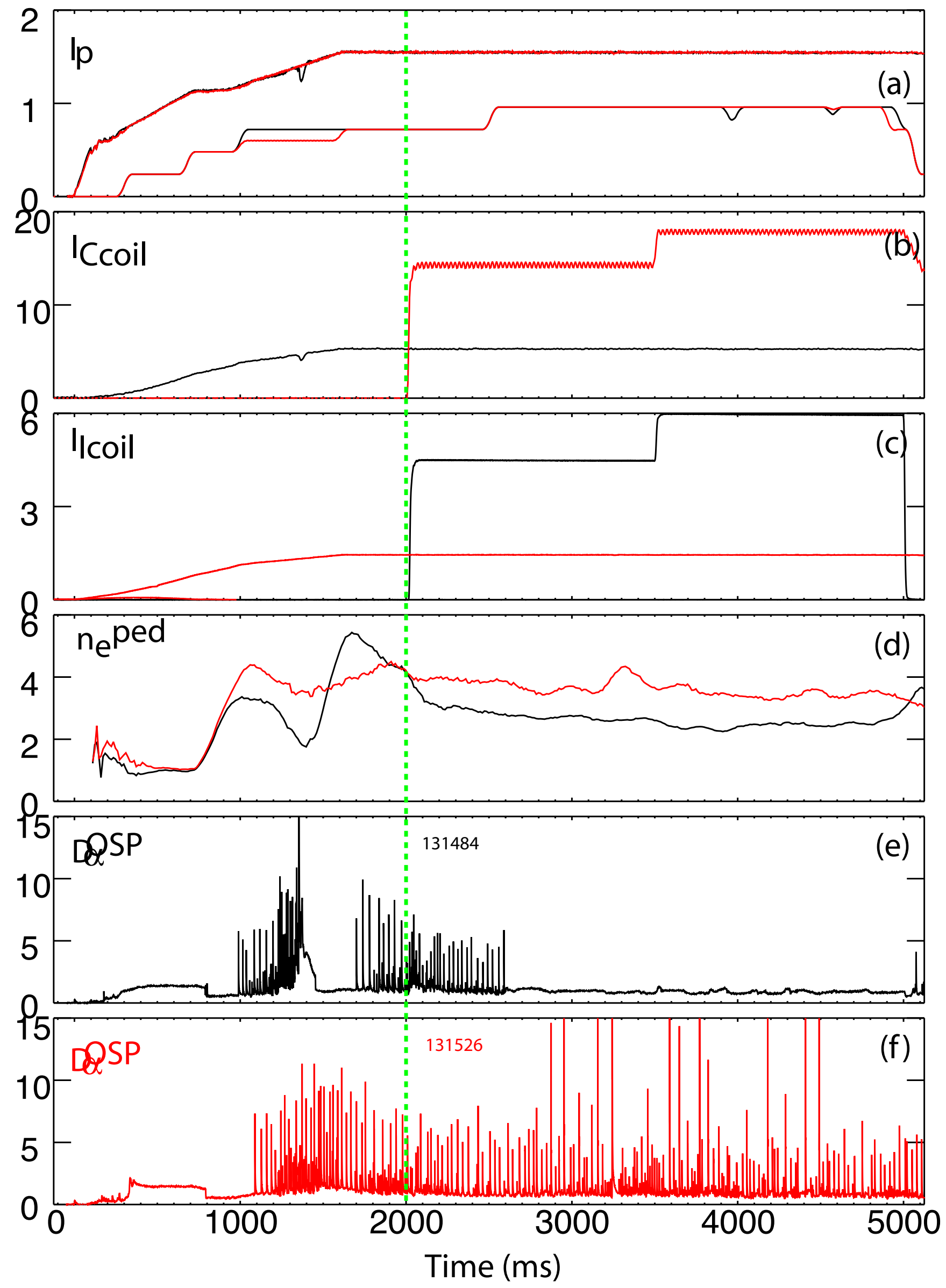

Fenstermacher_NF_Lett08_Fig.4 

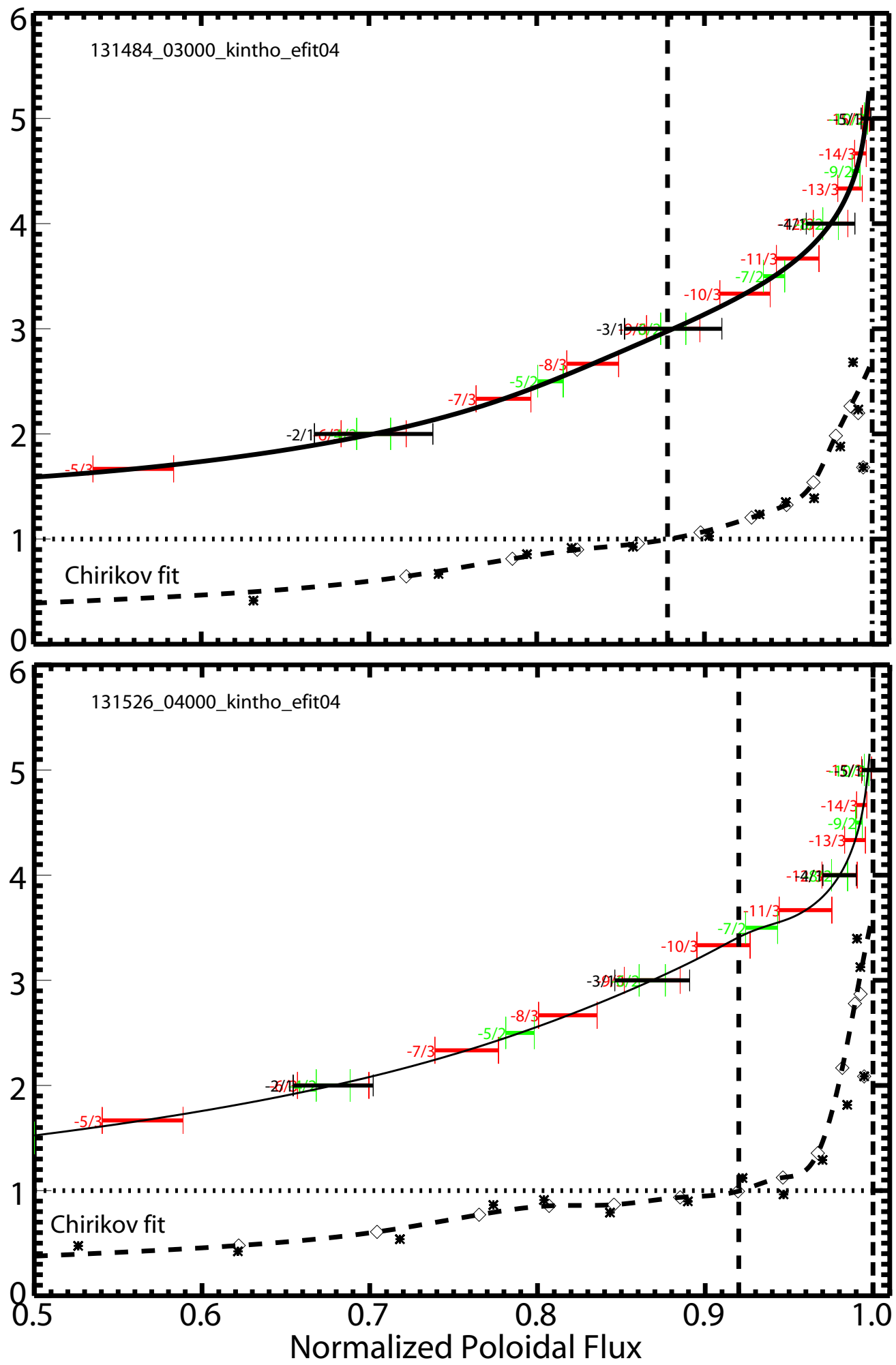

Fenstermacher_NF_Lett08_Fig.5 